\title{
Disentangling the effect of host-genotype and environment on the microbiome of the coral Acropora tenuis
}

\author{
Bettina Glasl ${ }^{\text {Corresp., }}$ 1,2,3 $^{\text {, Caitlin E Smith }}{ }^{1,2,3}$, David G Bourne ${ }^{1,2,3}$, Nicole S Webster ${ }^{1,3,4}$ \\ ${ }^{1}$ Australian Institute of Marine Science, Townsville, Queensland, Australia \\ 2 College of Science and Engineering, James Cook University, Townsville, Queensland, Australia \\ 3 AIMS@JCU, Townsville, Queensland, Australia \\ 4 Australian Centre for Ecogenomics, University of Queensland, Brisbane, Queensland, Australia \\ Corresponding Author: Bettina Glasl \\ Email address: b.glasl@aims.gov.au
}

Genotype-specific contributions to the environmental tolerance and disease susceptibility of corals are widely accepted. Yet our understanding of how host genotype influences the composition and stability of the coral microbiome subjected to environmental fluctuations is limited. To gain insight into the community dynamics and environmental stability of microbiomes associated with distinct coral genotypes, we assessed the microbial community associated with Acropora tenuis under single and cumulative pressure experiments. Experimental treatments comprised either a single pulse of reduced salinity (minimum of $28 \mathrm{psu}$ ) or exposure to the cumulative pressures of reduced salinity (minimum of $28 \mathrm{psu}$ ), elevated seawater temperature $\left(+2{ }^{\circ} \mathrm{C}\right)$, elevated $\mathrm{pCO}_{2}(900 \mathrm{ppm})$ and the presence of macroalgae. Analysis of $16 \mathrm{~S}$ rRNA gene amplicon sequence data revealed that $A$. tenuis microbiomes were highly host-genotype specific and maintained high compositional stability irrespective of experimental treatment. On average, $48 \%$ of the $A$. tenuis microbiome was dominated by Endozoicomonas. Amplicon sequence variants (ASVs) belonging to this genus were significantly different between host individuals. Although no signs of stress were evident in the coral holobiont and the vast majority of ASVs remained stable across treatments, a microbial indicator approach identified 26 ASVs belonging to Vibrionaceae, Rhodobacteraceae, Hahellaceae, Planctomycetes, Phylobacteriaceae, Flavobacteriaceae and Cryomorphaceae that were significantly enriched in corals exposed to single and cumulative stressors. While several recent studies have highlighted the efficacy of microbial indicators as sensitive markers for environmental disturbance, the high host-genotype specificity of coral microbiomes may limit their utility and we therefore recommend meticulous control of host-genotype effects in coral microbiome research. 


\section{Disentangling the effect of host-genotype and}

\section{2 environment on the microbiome of the coral Acropora}

\section{3 tenuis}

4

5 Bettina Glas $1^{1,2,3}$, Caitlin E. Smith ${ }^{1,2,3}$ David G. Bourne ${ }^{1,2,3}$ and Nicole S. Webster ${ }^{1,3,4}$

6

$7 \quad{ }^{1}$ Australian Institute of Marine Science, Townsville, Qld, Australia

$8{ }^{2}$ College of Science and Engineering, James Cook University, Townsville, Qld, Australia

93 AIMS@JCU, Townsville, Qld, Australia

${ }^{4}$ Australian Centre for Ecogenomics, University of Queensland, Brisbane, Qld, Australia

Corresponding Author:

Bettina Glasl $1^{1,2,3}$

PMB No 3, Townsville MC, Queensland, 4810, Australia 


\section{ABSTRACT}

36

37

38

\section{INTRODUCTION}

60 microbiome research.

Genotype-specific contributions to the environmental tolerance and disease susceptibility of corals are widely accepted. Yet our understanding of how host genotype influences the composition and stability of the coral microbiome subjected to environmental fluctuations is limited. To gain insight into the community dynamics and environmental stability of microbiomes associated with distinct coral genotypes, we assessed the microbial community associated with Acropora tenuis under single and cumulative pressure experiments.

Experimental treatments comprised either a single pulse of reduced salinity (minimum of $28 \mathrm{psu}$ ) or exposure to the cumulative pressures of reduced salinity (minimum of $28 \mathrm{psu}$ ), elevated seawater temperature $\left(+2{ }^{\circ} \mathrm{C}\right)$, elevated $p \mathrm{CO}_{2}(900 \mathrm{ppm})$ and the presence of macroalgae. Analysis of $16 \mathrm{~S}$ rRNA gene amplicon sequence data revealed that $A$. tenuis microbiomes were highly host-genotype specific and maintained high compositional stability irrespective of experimental treatment. On average, $48 \%$ of the $A$. tenuis microbiome was dominated by Endozoicomonas. Amplicon sequence variants (ASVs) belonging to this genus were significantly different between host individuals. Although no signs of stress were evident in the coral holobiont and the vast majority of ASVs remained stable across treatments, a microbial indicator approach identified 26 ASVs belonging to Vibrionaceae, Rhodobacteraceae, Hahellaceae, Planctomycetes, Phylobacteriaceae, Flavobacteriaceae and Cryomorphaceae that were significantly enriched in corals exposed to single and cumulative stressors. While several recent studies have highlighted the efficacy of microbial indicators as sensitive markers for environmental disturbance, the high host-genotype specificity of coral microbiomes may limit their utility and we therefore recommend meticulous control of host-genotype effects in coral

Corals contain abundant and diverse communities of microorganisms that together form a 1 holobiont (Rohwer et al. 2002). The photoautotrophic dinoflagellate endosymbionts of the family 2 Symbiodiniaceae are by far the best studied symbiotic partners of reef-building corals. 
63 Symbiodiniaceae lineages vary between coral species (Smith et al. 2017) and even between host 64 genotypes of conspecific corals (Brener-Raffalli et al. 2018). Fine-scale adaptations of the 65 Symbiodiniaceae lineages can influence the environmental sensitivity of their hosts (Baker 66 2003), as some Symbiodiniaceae lineages are more thermo-tolerant and hence infer higher 67 bleaching tolerance to corals (Rowan 2004). Corals also harbour diverse communities of 68 69 70 71 72 73 74 75 76 77 78 79 80 81 82 83 84 85 86 87 88 89 90 bacteria, archaea and viruses (Bourne et al. 2016; Hernandez-Agreda et al. 2017; Thurber et al. 2017). Excessive environmental stress resulting in coral bleaching, tissue necrosis and mortality, is often accompanied by a shift in the microbiome (Glasl et al. 2016; Zaneveld et al. 2017). While the importance of the microbiome to coral fitness is well appreciated (Bourne et al. 2016; Grottoli et al. 2018; Peixoto et al. 2017; Ziegler et al. 2017), the microbiome's potential to expand the environmental tolerance of coral holobionts via microbial shuffling and switching is far less understood (Webster \& Reusch 2017). Endozoicomonas, a bacterial genus commonly associated with marine invertebrates, is considered a putative symbiont of corals as it can occur at high abundance in aggregates within the tissue (Neave et al. 2016b) and loss of Endozoicomonas is frequently seen in bleached or diseased corals (Bayer et al. 2013b; Glasl et al. 2016). Pangenome analysis of Endozoicomonas has revealed evidence for functional specificity between strains (Neave et al. 2017), hence fine-scale changes in the composition or relative abundance of different Endozoicomonas strains may contribute to variation in the environmental tolerance and disease susceptibility of conspecific corals.

A fundamental question in microbiome research is whether host intrinsic factors (e.g. genetics) or the environment are the main drivers of microbiome composition and stability (Spor et al. 2011; Wullaert et al. 2018). The influence of host genetics and environmental factors on the community composition of a microbiome varies between host species and even between host compartments. For example, the rizhosphere microbiome of the perennial plant Boechera stricta are predominantly shaped by environmental factors, however, its leaf associated microbial community is largely controlled by host genetic factors (Wagner et al. 2016). Host-genotype specific factors also shape the gut microbiome of Drosophila melanogaster, a model system for animal-microbe interactions, and further mediate its nutritional phenotype (Chaston et al. 2016). While many coral microbiome studies have focused on the effect of environmental stress (e.g. elevated temperature, increased macroalgae abundance, anthropogenic pollution and declining water quality (Garren et al. 2009; Vega Thurber et al. 2009; Zaneveld et al. 2016; Zhang et al. 
94 2015)); the combined influence of host-genotype and environmental stress on the microbial 95 community composition remains largely unknown. This is a critical knowledge gap as microbiome-by-host genotype-by-environment interactions may have important implications for the resistance of corals to stress and disease. Considering the recent declines in coral reefs (De'ath et al. 2012; Hoegh-Guldberg et al. 2007; Hughes et al. 2017) and the key role microorganisms play in maintaining host health (Bourne et al. 2016), disentangling the effect of environment and host-genotype on a coral's microbiota is of utmost importance.

This study investigated the effect of host genotype-by-environment interactions on the microbiome of Acropora tenuis. The compositional variability of the A. tenuis microbiome associated with distinct host genotypes (individual coral colonies) was assessed with high taxonomic resolution based on amplicon sequence variants (ASV). The stability of the microbiome was further investigated by exposing corals to acute salinity fluctuations (ranging from 35 psu to $28 \mathrm{psu}$ ) under current (sea surface temperature of $27.5^{\circ} \mathrm{C}$ and $p \mathrm{CO}_{2}$ of $400 \mathrm{ppm}$ ) and future (sea surface temperature of $29.5^{\circ} \mathrm{C}, p \mathrm{CO}_{2}$ of $900 \mathrm{ppm}$ and macroalgae) projected reef conditions. Stress treatments were designed to simulate environmental conditions that $A$. tenuis can experience in their natural environment. Both stress treatments (single and cumulative stress) consisted of a non-lethal low salinity pulse, mimicking freshwater influx into the reef as occurs after large rainfall events, often linked to cyclones that cross the Eastern Australian coastline and result in large riverine flows into the nearshore and mid-shelf reef areas of the GBR (e.g. Jones \& Berkelmans 2014; VanWoesik et al. 1995).

\section{MATERIALS \& METHODS}

\section{Coral colony collection and experimental design}

Nine Acropora tenuis colonies were collected from Davies Reef (Great Barrier Reef, Australia) in March 2017 and transported to the National SeaSimulator at the Australian Institute of Marine Science (Townsville, Australia). Corals were fragmented into coral nubbins, glued onto aragonite plugs and kept at control temperature $\left(27.5^{\circ} \mathrm{C}\right)$ and light $\left(150 \mathrm{~mol}\right.$ photons $\left.\mathrm{m}^{-2} \mathrm{~s}^{-1}\right)$ conditions in indoor flow-through aquaria for three weeks to allow healing. Corals were collected under the permit G12/35236.1 granted by the Great Barrier Reef Marine Park Authority to the Australian Institute of Marine Science. 
The experimental design consisted of three treatment conditions: 1) control, 2) single

125

126

127

128

129

130

131

132

133

134

135

136

137

138

139

140

141

142

143

144

145

146

147

148

149

150

151

152

153

154 stress and 3) cumulative stress treatment (Fig 1). Nubbins of all nine A. tenuis genotypes (A-I) were exposed to all three treatment conditions to explore microbiome variation according to host genotype. Each experimental aquarium (three aquaria per treatment) held nubbins of three $A$. tenuis genotypes (four nubbins per genotype, total of 12 nubbins per aquarium). Coral nubbins were acclimated to experimental aquaria for three weeks during which corals in the cumulative stress treatment were gradually ramped to $29.5^{\circ} \mathrm{C}$ and $900 \mathrm{ppm} p \mathrm{CO}_{2}$ over a period of 12 days. Corals in the control and single stressor treatments were kept at stable temperature $\left(27.5^{\circ} \mathrm{C}\right)$ and ambient (400 ppm) $\mathrm{pCO}_{2}$ conditions throughout the experiment.

Salinity was ramped down over $3 \mathrm{~h}$ to a minimum of $28 \mathrm{psu}$ and oscillated between 28 psu and 30 psu in a six-hour rhythm to simulate natural fluctuations occurring on reefs (tidal influences $)$. Temperature and $p \mathrm{CO} 2$ adjusted freshwater $(0.2 \mu \mathrm{m}$ filtered $)$ was used to lower salinities prior to supplying the low saline seawater to the aquaria tanks. After seven days of low salinity, the salinity was ramped up $(3 \mathrm{~h})$ to $35 \mathrm{psu}$. In the cumulative stress treatment, corals were additionally exposed to elevated temperature $\left(29.5^{\circ} \mathrm{C}\right), \mathrm{CO}_{2}(900 \mathrm{ppm})$ and macroalgae (Sargassum sp.), as predicted for the end of the $21^{\text {st }}$ century (IPCC 2014).

Samples were collected regularly throughout the experiment (see Fig 1), including $24 \mathrm{~h}$ before the salinity pulse was induced (day 1) and at three time points (day 10, day 14 and day 19) after the low-salinity stress exposure. All nubbins were processed as follows: effective quantum yield was measured (pulse amplitude modulation fluorometry), photographed, inspected for visual signs of stress (tissue lesions, bleaching and necrosis), rinsed with $0.2 \mu \mathrm{m}$ filter-sterilized seawater, snap frozen in liquid nitrogen and stored at $-80{ }^{\circ} \mathrm{C}$ until further processing. Coral nubbins were defrosted on ice before tissue was removed with an airgun in $1 \mathrm{x}$ PBS $(\mathrm{pH}=7.4)$, homogenised for $1 \mathrm{~min}$ at $12.5 \mathrm{rpm}$ with a hand-held tissue homogeniser (Heidolph Silent Crusher M) and subsequently aliquoted for the quantification of Symbiodiniaceae cell density, chlorophyll $a$, protein concentration and DNA extraction for amplicon-based sequencing of the 16S rRNA gene. Aliquots $(500 \mu \mathrm{l})$ for Symbiodiniaceae cell counts were fixed with formaldehyde (final concentration $1.5 \%$ ) and stored in the dark at room temperature. Aliquots for chlorophyll $a$, protein and DNA extraction ( $1 \mathrm{ml}$ each) were centrifuged for $10 \mathrm{~min}$ at 16,000 $\mathrm{g}$, the supernatant was discarded and the remaining pellet was snap frozen in liquid nitrogen and stored at $-80{ }^{\circ} \mathrm{C}$ until further processing. Coral nubbin surface area was assessed by a single 
155 paraffin wax dipping for $2 \mathrm{~s}$ followed by $5 \mathrm{~min}$ air-drying. The weight of each coral nubbin 156 before and after dipping was quantified and the surface area was calculated against a standard 157 curve.

158

159

160

161

162

163

164

165

166

167

168

169

170

171

172

173

174

175

176

177

178

179

180

181

182

183

184

185

\section{Physiology of Symbiodiniaceae and the coral holobiont}

The effective quantum yield of the Symbiodiniaceae was measured using pulse amplitude modulation (PAM) fluorometry. Corals were light adapted ( $5 \mathrm{~h}$ ) before measuring the response of the photosystem II effective quantum yield ( $\Delta \mathrm{F} / \mathrm{Fm}$ ') with a Heinz Walz ${ }^{\mathrm{TM}}$ Imaging PAM as previously described (Chakravarti et al. 2017). Coral nubbins were exposed to a saturation pulse and the minimum and maximum fluorescence was recorded and effective quantum yield was calculated (see Equation S1 ).

Symbiodiniaceae cell densities were manually counted under a stereomicroscope using formaldehyde fixed tissue samples (final $\mathrm{c}=1.5 \%$ ). Samples were briefly vortexed and $9 \mu \mathrm{l}$ of each sample was added to either side of two haemocytometers and the density of symbiont cells was quantitatively normalised to the tissue blastate and aliquot volume, and standardised to the nubbin's surface area.

Chlorophyll $a$ was extracted and concentrations were measured using a spectrophotometric assay. Tissue pellets were defrosted on ice, centrifuged at 16,000 g for $10 \mathrm{~min}$ at $4{ }^{\circ} \mathrm{C}$, and remaining supernatant was discarded. Pellets were re-suspended in $1 \mathrm{ml}$ of $100 \%$ acetone and incubated in the dark for $24 \mathrm{~h}$ at $4{ }^{\circ} \mathrm{C}$ after which they were centrifuged at $16,000 \mathrm{~g}$ for $10 \mathrm{~min}$ and supernatant $(200 \mu \mathrm{l})$ was pipetted into a 96-well plate in triplicate. Absorbance at $630 \mathrm{~nm}$ and $663 \mathrm{~nm}$ was measured using a BioTek ${ }^{\mathrm{TM}}$ microplate reader and chlorophyll a concentration was calculated (see S1 Equation), quantitatively normalised to the tissue blastate and aliquot volume, and standardised to the nubbin's surface area.

Total protein was quantified using a Pierce ${ }^{\mathrm{TM}}$ BCA Protein Assay Kit (Thermo Scientific) following the manufacturer's instruction. Absorbance was measured in triplicate for each sample at $562 \mathrm{~nm}$ in a BioTek ${ }^{\mathrm{TM}}$ Plate reader. Standard curves were calculated using a bovine serum albumin (BSA) solution, creating a working range between 20 and $2000 \mu \mathrm{g} \mathrm{ml}^{-1}$ and total protein was calculated against the BSA standard curve, quantitatively normalised to the tissue blastate and aliquot volume, and standardised to the surface area of each individual nubbin. 
186 DNA extraction, 16S rRNA gene sequencing and analysis

187 DNA of all coral samples was extracted using the DNeasy PowerBiofilm Kit (QIAGEN)

188 following the manufacturer's instructions. Blank extractions were included to control for kit

189 contamination. Coral DNA extracts were stored at $-80^{\circ} \mathrm{C}$ until shipment on dry ice to Ramaciotti

190 Centre (University of New south Wales, Australia) for sequencing. The V1-V3 region of the 16S

191 rRNA gene was amplified using primers 27F (5 - AGAGTTTGATCMTGGCTCAG -3`; Lane

192 1991) and 519R (5'-GWATTACCGCGGCKGCTG -3`; Turner et al. 1999) and libraries were

193 prepared with the Illumina TruSeq protocol, followed by Illumina MiSeq 2 x 300 bp sequencing

194 (see Table S1).

195 Demultiplexed paired end reads were analyzed in QIIME2 (Version 2017.9.0;

196 https://qiime2.org) as previously described by Glasl et al. (2018). In brief, forward and reverse

197 reads were truncated at their 3' end at the 296 and 252 sequencing positions, respectively.

198 Samples were checked for chimeras and grouped into features based on $100 \%$ sequence

199 similarity, from here on referred to as ASV (amplicon sequence variants), using DADA2

200 (Callahan et al. 2016). Multiple de novo sequence alignments of the representative sequences

201 were performed using MAFFT (Katoh et al. 2002). Non-conserved and highly gapped columns

202 from the alignment were removed using default settings of the mask option in QIIME2.

203 Unrooted and rooted trees were generated for phylogenetic diversity analysis using FastTree. For

204 taxonomic assignment, a Naïve-Bayes classifier was trained on the SILVA v123 99 \%

205 Operational Taxonomic Units, where reference sequences only included the V1-V2 regions

$206(27 \mathrm{~F} / 519 \mathrm{R}$ primer pair) of the 16S rRNA genes. The trained classifier was applied to the

207 representative sequences to assign taxonomy. A total of 11,063,364 reads were retrieved from 100

208 sequenced samples and clustered into 4624 ASVs (Table 1). Chloroplast and Mitochondria derived

209 sequence reads and singletons were removed from the dataset and the feature table was rarefied

210 to an even sequencing depth of 3,506 sequencing reads, leading to the exclusion of four samples.

211 Demultiplexed sequences and metadata are available from the NCBI Sequence Read Archives

212 (SRA) under accession number PRJNA492377.

213

214 Statistical analysis

215 Statistical analysis was performed in R (R Development Core Team 2008). Holobiont health

216 metadata were z-score standardized and variation between treatments and host genotypes was 
217 evaluated using Analysis of Variance (ANOVA) and if applicable, variations were further 218 assessed with a Tukey post-hoc test. Multivariate statistical approaches including Multivariate 219 Homogeneity of Group Dispersion ('vegan package' (Oksanen et al. 2013)), Permutation 220 Multivariate Analysis of Variance (PERMANOVA, 'vegan package' (Oksanen et al. 2013)), 221 Non-metric multidimensional scaling (NMDS ‘phyloseq package’, (McMurdie \& Holmes 2013)) 222 and distance based Redundancy Analysis (db-RDA 'phyloseq package' (McMurdie \& Holmes 223 2013)) were based on Bray Curtis dissimilarities. Mantel statistics based on Pearson's product224 moment correlation (mantel test, 'vegan package' (Oksanen et al. 2013) were used to evaluate 225 whether sample-to-sample dissimilarities in microbiome composition and physiological 226 holobiont health parameters (protein concentration, chlorophyll $a$ concentration,

227 Symbiodiniaceae cell densities and effective quantum yield) were correlated. Holobiont health 228 parameters were z-score standardised and dissimilarity matrices were based on Bray Curtis 229 dissimilarities.

Alpha diversity measures including richness and Shannon diversity for the Endozoicomonas community were analyzed using the 'phyloseq package' (McMurdie \& Holmes 2013). Variation in the total relative abundance of all Endozoicomonas ASVs per sample between treatments, over time and between host-genotypes was assessed using ANOVAs with arcsine-square-root transformed relative abundance data. The phylogenetic tree of the 11 most abundant Endozoicomonas ASVs was produced with phyloseq (McMurdie \& Holmes 2013) using the Newick rooted tree generated in QIIME 2 (Version 2017.9.0; https://qiime2.org). was used to identify ASVs significantly associated with treatment groups (control, single stress and cumulative stress) based on their occurrence and abundance distribution. Day 1 samples were excluded from the IndVal analysis to restrict the dataset to ASVs significantly associated with coral tissue after stress exposure (day 10, day 14 and day 19). (McMurdie \& Holmes 2013). Alluvial diagram was generated in RAWGraph (Mauri et al. 2017).

\section{Coral holobiont physiological response}


247 Corals showed no visual signs of stress (change in pigmentation, bleaching, tissue necrosis

248 and/or mortality) in any treatment. Chlorophyll $a$ concentrations remained stable between

249 treatments (one-way ANOVA with sampling time point as blocking factor, $\mathrm{F}_{(2 / 94)}=2.707, \mathrm{p}=$

250 0.072), however, effective quantum yield ( $\Delta \mathrm{F} / \mathrm{Fm}$ '; one-way ANOVA with sampling time point

251 as blocking factor, $\mathrm{F}_{(2 / 94)}=15.52, \mathrm{p}=1.49 \times 10^{-6}$ ), symbiont cell densities (one-way ANOVA

252 with sampling time point as blocking factor, $\left.\mathrm{F}_{(2 / 94)}=8.83, \mathrm{p}=3.06 \times 10^{-4}\right)$ and protein

253 concentration (one-way ANOVA with sampling time point as blocking factor, $\mathrm{F}_{(2 / 94)}=5.563, \mathrm{p}=$

$2545.21 \times 10^{-3}$ ) varied significantly between treatments within sampling time points (Fig 2A). Coral

255 nubbins in the cumulative stress treatment contained significantly lower protein and symbiont

256 cell densities, while displaying significantly higher effective quantum yield compared to nubbins

257 in the control and single stressor treatments (Tukey Posthoc test, Table S2). Furthermore,

258 effective quantum yield (one-way ANOVA, $\mathrm{F}_{(8 / 91)}=2.688, \mathrm{p}=0.0106$ ), symbiont cell densities

259 (one-way ANOVA, $\mathrm{F}_{(8 / 91)}=4.334, \mathrm{p}=1.86 \times 10^{-4}$ ) and chlorophyll $a$ concentrations (one-way

260 ANOVA, $\mathrm{F}_{(8 / 91)}=2.773, \mathrm{p}=8.64 \times 10^{-3}$ ) varied significantly between host genotypes (Fig $\left.2 \mathrm{~B}\right)$.

261 Protein concentration, however, was unaffected by host genotype (one-way ANOVA, $\mathrm{F}_{(8 / 91)}=$

$2621.783, \mathrm{p}=0.0906$ ) and hence was the only holobiont health parameter solely affected by

263 treatment.

264

265 Microbial community response

266 The microbiome of $A$. tenuis remained highly stable across treatments, with no significant

267 changes in the heterogeneity, also referred to as multivariate dispersion (one-way ANOVA,

$268 \mathrm{~F}_{(2 / 93)}=1.2107, \mathrm{p}=0.3026$; Fig $3 \mathrm{~A}$ ), or in community composition (PERMANOVA, $\mathrm{p}=0.5156$,

269 10,000 permutations; Fig 3B). However, the microbiome composition varied significantly

270 between individual host genotypes (PERMANOVA, $\mathrm{p}=9.99 \times 10^{-5}, 10,000$ permutations), but

271 was unaffected by treatment, sampling time point or tank effects when tested for each genotype

272 individually (PERMANOVA with host-genotype as blocking factor, 10,000 permutations, Table

273 S3). Similar results were obtained using presence/absence data (Fig S1). Host genotype was the

274 only significant factor, explaining $32.4 \%$ of the observed community variation (permutational

275 ANOVA for db-RDA based on 1,000 permutations, $\mathrm{p}=9.99 \times 10^{-4}$; Fig S2). Treatment and

276 holobiont health parameters did not significantly contribute to the microbiome variation (Table

277 S4). Furthermore, no significant correlation between similarity matrices based on microbiome 
278 composition and physiological holobiont health parameters was observed (chlorophyll $a$, protein, 279 effective quantum yield and symbiont cell density; Mantel statistic based on Pearson's product280 moment correlation $\mathrm{r}=-0.0238, \mathrm{p}=0.6243,10,000$ permutations).

281

\section{Endozoicomonas assemblage}

283 Endozoicomonas affiliated sequences comprised the majority of the A. tenuis microbiome, 284 representing $48 \%$ ( $\pm 29 \%$ ) of the community (based on proportion of reads) and comprising 133 285 unique ASVs. One Endozoicomonas strain (ASV 11) was consistently present (100\% of all 286 samples) and highly abundant (19\% $\pm 12 \%)$ throughout the experiment (Fig 4). The A. tenuis 287 microbiome also contained diverse bacteria affiliated with phyla including Proteobacteria (30 $288 \%$ ), Actinobacteria (10 \%), Firmicutes (2.4\%) and Bacteroidetes (1.9\%; Fig 4).

The total relative abundance of Endozoicomonas was not affected by treatment (one-way ANOVA, $F_{(2 / 84)}=0.473, p=0.625$ ), sampling time point (one-way ANOVA, $F_{(3 / 84)}=0.588, p=$ 0.625 ) or the interaction of treatment-by-sampling time point (one-way ANOVA, $\mathrm{F}_{(6 / 84)}=0.696$, $\mathrm{p}=0.654)$. However, total relative Endozoicomonas abundance varied significantly between host genotypes (one-way ANOVA, $\mathrm{F}_{(8 / 87)}=3.741, \mathrm{p}=2.04 \times 10^{-4}$ ) and remained stable between treatments when tested for each genotype individually (within subject ANOVA, $\mathrm{F}_{(2 / 85)}=0.756, \mathrm{p}$ $295=0.473$ ); Fig 5A). genotypes (PERMANOVA, p = $9.99 \times 10^{-5}, 10,000$ permutations, Fig 5), however, was unaffected by treatment, sampling time point or tank (PERMANOVA with host-genotype as blocking factor, 10,000 permutations, Table S5). Furthermore, host-genotype significantly explained $26.4 \%$ of the observed compositional variability of the Endozoicomonas community (permutational ANOVA for db-RDA based on 1,000 permutations, $\mathrm{p}=9.99 \times 10^{-5}$; Fig 5B).

302

\section{Microbial indicators for environmental stress}

Indicator value analysis was performed to assess if specific ASVs could be identified as indicators for environmental stress treatments. Despite the vast majority of ASVs (i.e. 4598 ASVs) showing no response to experimental treatment, 26 ASVs were significantly associated (p $<0.05$ ) with one and / or two treatment groups (Fig 6, Table S6). The identified indicator ASVs 
309 Endozoicomonas), Planctomycetes, Phylobacteriaceae, Flavobacteriaceae and Cryomorphaceae 310 (Fig 6).

311

\section{DISCUSSION}

313 Elucidating the effect of host genotype on microbiome composition and understanding

314 consequences of environmental change for holobiont stability is central to predicting the

315 influence of host genetics on the stress tolerance of corals. Here we followed the compositional

316 stability of microbiomes associated with nine distinct $A$. tenuis genotypes when exposed to

317 control, single and cumulative stress treatments over time. The $A$. tenuis microbiome varied

318 significantly between coral genotypes, with host genotype being a much stronger driver of

319 microbiome variation than environment. Similar host-genotype specificities have recently been

320 described for sponge microbiomes (Glasl et al. 2018) and are also frequently reported for plant,

321 crustacean and human microbiomes (Balint et al. 2013; Macke et al. 2017; Spor et al. 2011).

322 Traditional coral health parameters targeting the coral algal symbiont (i.e. chlorophyll $a$

323 concentrations, symbiont cell densities, effective quantum yield) were also significantly affected

324 by host-genotype, although no correlation between these parameters and the microbiome was

325 observed. This suggests that the A. tenuis microbiome composition remains largely unaffected by

326 the performance and density of the algal symbiont, and that other host intrinsic factors (e.g.

327

328

329

330

331

332

333

334

335

336

337

338

339

genetics) and/or the environmental life-history of individual genotypes fine-tune the microbiome composition.

Endozoicomonas form symbiotic partnerships with diverse marine invertebrates (Neave et al. 2016a). In corals, Endozoicomonas occur as dense clusters within the coral tissue and in some bacterial 16S rRNA gene profiling studies they can reach relative abundances as high as 95\% of retrieved sequences (Bayer et al. 2013a; Neave et al. 2016a; Pogoreutz et al. 2018). Loss of Endozoicomonas from the coral microbiome has been correlated with negative health outcomes for the coral host, though their direct effects on host fitness are unknown (Bourne et al. 2008; Glasl et al. 2016; Ziegler et al. 2016). In A. tenuis, we detected no significant change in the relative frequency, alpha diversity, richness and community composition of Endozoicomonas following exposure to non-lethal environmental stress. These results are consistent with findings for Pocillopora verrucosa, where Endozoicomonas remains the dominant symbiont even under bleaching conditions (Pogoreutz et al. 2018). In our study, the Endozoicomonas community 
340 generally exhibited high host-genotype specificity at the ASV level, though a single

341 Endozoicomonas strain (ASV 11) was consistently shared among all coral nubbins and

342 genotypes (including field control samples - data not shown). This ubiquitous strain likely

343 represents a stable and consistent member of the resident Endozoicomonas community. A stable

344 core is often described as a key feature of a symbiotic coral microbiome (Ainsworth et al. 2015;

345 Hernandez-Agreda et al. 2017), and despite being ubiquitously persistent between conspecific

346 corals, the core characteristically only comprises a few members of the whole microbiome

347 (Hernandez-Agreda et al. 2018).

348 While the Endozoicomonas community as a whole was not significantly affected by 349 environmental treatment, one Endozoicomonas ASV was identified as a significant indicator for 350 environmental stress. Similar environmental sensitivity has been reported for two prevalent 351 Endozoicomonas species following exposure to elevated dissolved organic carbon (Pogoreutz et 352 al. 2018). Although these Endozoicomonas affiliated ASVs show high sequence identity, small 353 variations in the rRNA gene sequence can impact the biology and pathogenicity of bacteria (Cilia 354 et al. 1996; Fukushima et al. 2002), hence single nucleotide variations (ASV level) may affect 355 the functional role of microbes with flow on consequences for the coral holobiont. Shuffling and 356 switching of Endozoicomonas strains may therefore provide the coral holobiont with an enhanced capacity to cope with shifting environmental conditions (Neave et al. 2017), although characterisation of the symbiotic contribution made by Endozoicomonas to the coral host is

359 360 361 362 363 364 365 required to better understand the ecological significance of these findings.

Recent studies have highlighted the potential for coral microbiomes to act as sensitive markers for environmental disturbance (Glasl et al. 2017; Roitman et al. 2018). Here we showed that a small number of ASVs, including taxa commonly reported to increase under host stress (i.e. Vibrionaceae, Rhodobacteraceae (Ben-Haim et al. 2003; Bourne et al. 2016; Sunagawa et al. 2010)), were significantly associated with the tissue of $A$. tenuis exposed to single and cumulative stress treatments. However, despite the potential diagnostic value of these ASVs, host genotype overwhelmed any overarching effect of environment on the coral microbiome. This high divergence in the microbiome between conspecific corals is likely to hinder our ability to detect fine-scale variation of sensitive microbial indicator taxa. Therefore, unless hostgenotype independent microbial indicators can be identified and validated, the efficacy of 
370 integrating microbial community data into coral health monitoring initiatives appears unfeasible

371 due to high compositional variability between microbiomes of conspecific corals.

372

\section{CONCLUSIONS}

374 This study shows that the $A$. tenuis microbiome varies significantly between host individuals

375 (genotypes) and that these genotype-specific communities persist during exposure to non-lethal

376

377

378

379

380

381

382

383

384

385

386

387

388

389

390

391

392

393

394

395

396

397

398

399

400

401

402

403

404

environmental disturbances. Consideration of microbiome-by-host genotype-by-environment

effects is therefore needed to elucidate how intraspecific variations of the microbiome affect the susceptibility of corals to environmental stress and disease. Furthermore, microbial variability between individual coral genotypes may cloud our ability to identify universal microbial changes during periods of adverse environmental conditions. This is particularly relevant if establishing sensitive microbial indicators for sub-lethal environmental disturbances (tested in this study), since the observed stability of the coral microbiome combined with the host genotype specificity likely precludes the robust assignment of microbial indicators across broad scales.

\section{ACKNOWLEDGEMENTS}

We thank Victoria Lydick and Andrew Ball for their help during the experiment. We also acknowledge the technical support provided by the National SeaSimulator, and are particularly grateful to Craig Humphrey and Andrea Severati for their support with the experimental system. We thank Pedro R. Frade for inspiring scientific discussions on the coral microbiome and photobiology of corals and Jonathan Barton for his insights into coral husbandry.

\section{REFERENCES}

Ainsworth TD, Krause L, Bridge T, Torda G, Raina J-B, Zakrzewski M, Gates RD, Padilla-Gamino JL, Spalding HL, Smith C, Woolsey ES, Bourne DG, Bongaerts P, Hoegh-Guldberg O, and Leggat W. 2015. The coral core microbiome identifies rare bacterial taxa as ubiquitous endosymbionts. ISME J. 9:2261-2274.

Baker AC. 2003. Flexibility and specificity in coral-algal symbiosis: Diversity, ecology, and biogeography of Symbiodinium. Annual Review of Ecology Evolution and Systematics 34:661689.

Balint M, Tiffin P, Hallstrom B, O'Hara RB, Olson MS, Fankhauser JD, Piepenbring M, and Schmitt I. 2013. Host genotype shapes the foliar fungal microbiome of balsam poplar (Populus balsamifera). PLoS ONE 8:e53987.

Bayer T, Neave MJ, Alsheikh-Hussain A, Aranda M, Yum LK, Mincer T, Hughen K, Apprill A, and Voolstra CR. 2013. The Microbiome of the Red Sea coral Stylophora pistillata is dominated by 
405

406

407

408

409

410

411

412

413

414

415

416

417

418

419

420

421

422

423

424

425

426

427

428

429

430

431

432

433

434

435

436

437

438

439

440

441

442

443

444

445

446

447

448

449

450

451

452

453

454

tissue-associated Endozoicomonas bacteria. Applied and Environmental Microbiology 79:47594762.

Ben-Haim Y, Thompson F, Thompson C, Cnockaert M, Hoste B, Swings J, and Rosenberg E. 2003. Vibrio coralliilyticus sp. nov., a temperature-dependent pathogen of the coral Pocillopora damicornis. International Journal of Systematic and Evolutionary Microbiology 53:309-315.

Bourne DG, Iida Y, Uthicke S, and Smith-Keune C. 2008. Changes in coral-associated microbial communities during a bleaching event. ISME J 2:350-363.

Bourne DG, Morrow KM, and Webster NS. 2016. Coral Holobionts: Insights into the coral microbiome: Underpinning the health and resilience of reef ecosystems. Annual Reviews of Microbiology 70:317-340.

Brener-Raffalli K, Clerissi C, Vidal-Dupiol J, Adjeroud M, Bonhomme F, Pratlong M, Aurelle D, Mitta G, and Toulza E. 2018. Thermal regime and host clade, rather than geography, drive

Symbiodinium and bacterial assemblages in the scleractinian coral Pocillopora damicornis sensu lato. Microbiome 6:39.

Callahan BJ, McMurdie PJ, Rosen MJ, Han AW, Johnson AJA, and Holmes SP. 2016. DADA2: Highresolution sample inference from Illumina amplicon data. Nature Methods 13:581.

Chakravarti LJ, Beltran VH, and van Oppen MJH. 2017. Rapid thermal adaptation in photosymbionts of reef-building corals. Glob Chang Biol 23:4675-4688.

Chaston JM, Dobson AJ, Newell PD, and Douglas AE. 2016. Host Genetic Control of the Microbiota Mediates the Drosophila Nutritional Phenotype. Applied and Environmental Microbiology 82:671-679.

Cilia V, Lafay B, and Christen R. 1996. Sequence heterogeneities among 16S ribosomal RNA sequences, and their effect on phylogenetic analyses at the species level. Molecular Biology and Evolution 13:451-461.

De Cáceres M, and Legendre P. 2009. Associations between species and groups of sites: indices and statistical inference. Ecology 90:3566-3574.

De'ath G, Fabricius KE, Sweatman H, and Puotinen M. 2012. The 27-year decline of coral cover on the Great Barrier Reef and its causes. Proceedings of the National Academy of Sciences of the United States of America 109:17995-17999.

Fukushima M, Kakinuma K, and Kawaguchi R. 2002. Phylogenetic analysis of Salmonella, Shigella, and Escherichia coli strains on the basis of the gyrB gene sequence. Journal of Clinical Microbiology 40:2779-2785.

Garren M, Raymundo L, Guest J, Harvell CD, and Azam F. 2009. Resilience of coral-associated bacterial communities exposed to fish farm effluent. PLoS ONE 4:e7319.

Glasl B, Herndl GJ, and Frade PR. 2016. The microbiome of coral surface mucus has a key role in mediating holobiont health and survival upon disturbance. ISME J 10:2280-92

Glasl B, Smith CE, Bourne DG, and Webster NS. 2018. Exploring the diversity-stability paradigm using sponge microbial communities. Scientific Reports 8:8425.

Glasl B, Webster NS, and Bourne DG. 2017. Microbial indicators as a diagnostic tool for assessing water quality and climate stress in coral reef ecosystems. Marine Biology 164:91

Grottoli AG, Martins PD, Wilkins MJ, Johnston MD, Warner ME, Cai WJ, Melman TF, Hoadley KD, Pettay DT, Levas S, and Schoepf V. 2018. Coral physiology and microbiome dynamics under combined warming and ocean acidification. PLOS ONE 13:e191156.

10.1371/journal.pone.0191156

Hernandez-Agreda A, Gates RD, and Ainsworth TD. 2017. Defining the core microbiome in corals' microbial soup. Trends in Microbiology 25:125-140.

Hernandez-Agreda A, Leggat W, Bongaerts P, Herrera C, and Ainsworth TD. 2018. Rethinking the coral microbiome: simplicity exists within a diverse microbial biosphere. MBio 9:e00812-00818.

Hoegh-Guldberg O, Mumby PJ, Hooten AJ, Steneck RS, Greenfield P, Gomez E, Harvell CD, Sale PF, Edwards AJ, Caldeira K, Knowlton N, Eakin CM, Iglesias-Prieto R, Muthiga N, Bradbury RH, 
455

456

457

458

459

460

461

462

463

464

465

466

467

468

469

470

471

472

473

474

475

476

477

478

479

480

481

482

483

484

485

486

487

488

489

490

491

492

493

494

495

496

497

498

499

500

501

502

503

504

505

Dubi A, and Hatziolos ME. 2007. Coral reefs under rapid climate change and ocean acidification. Science 318:1737-1742.

Hughes TP, Barnes ML, Bellwood DR, Cinner JE, Cumming GS, Jackson JBC, Kleypas J, van de Leemput IA, Lough JM, Morrison TH, Palumbi SR, van Nes EH, and Scheffer M. 2017. Coral reefs in the Anthropocene. Nature 546:82-90.

IPCC 2014. Climate Change 2014: Synthesis Report. Contribution of Working Groups I, II and III to the Fifth Assessment Report of the Intergovernmental Panel on Climate Change. In: R.K. Pachauri, and Meyer LA, editors. IPCC. Geneva, Switzerland.

Jones AM, and Berkelmans R. 2014. Flood impacts in Keppel Bay, southern Great Barrier Reef in the aftermath of cyclonic rainfall. PLoS ONE 9:e84739.

Katoh K, Misawa K, Kuma K, and Miyata T. 2002. MAFFT: a novel method for rapid multiple sequence alignment based on fast Fourier transform. Nucleic Acids Research 30:3059-3066.

Lane DJ. 1991. 16S/23S rRNA sequencing. In: Stackebrandt E, and Goodfellow M, eds. Nucleic acid techniques in bacterial systematics. New York: John Wiley and Sons, 115-175.

Macke E, Callens M, De Meester L, and Decaestecker E. 2017. Host-genotype dependent gut microbiota drives zooplankton tolerance to toxic cyanobacteria. Nature Communications 8:1608.

Mauri M, Elli T, Caviglia G, Uboldi G, and Azzi M. 2017. RAWGraphs: A visualisation platform to create open outputs. New York, NY, USA.

McMurdie PJ, and Holmes S. 2013. phyloseq: an R package for reproducible interactive analysis and graphics of microbiome census data. PLoS ONE 8:e61217.

Neave MJ, Apprill A, Ferrier-Pages C, and Voolstra CR. 2016a. Diversity and function of prevalent symbiotic marine bacteria in the genus Endozoicomonas. Applied Microbiology and Biotechnology 100:8315-8324.

Neave MJ, Michell CT, Apprill A, and Voolstra CR. 2017. Endozoicomonas genomes reveal functional adaptation and plasticity in bacterial strains symbiotically associated with diverse marine hosts. Scientific Reports 7:40579.

Neave MJ, Rachmawati R, Xun L, Michell CT, Bourne DG, Apprill A, and Voolstra CR. 2016b. Differential specificity between closely related corals and abundant Endozoicomonas endosymbionts across global scales. ISME J. 11:186-200

Oksanen J, Blanchet FG, Kindt R, Legendre P, Minchin PR, O'Hara RB, Simpson GL, Solymos P, Stevens MHH, and Wagner H. 2013. vegan: Community Ecology Package. $R$ package version 20-9.

Peixoto RS, Rosado PM, Leite DCD, Rosado AS, and Bourne DG. 2017. Beneficial Microorganisms for Corals (BMC): Proposed mechanisms for coral health and resilience. Frontiers in Microbiology $8: 341$.

Pogoreutz C, Radecker N, Cardenas A, Gardes A, Wild C, and Voolstra CR. 2018. Dominance of Endozoicomonas bacteria throughout coral bleaching and mortality suggests structural inflexibility of the Pocillopora verrucosa microbiome. Ecology and Evolution 8:2240-2252.

R Development Core Team. 2008. R: A language and environment for statistical computing. $R$ Foundation for Statistical Computing.

Rohwer F, Seguritan V, Azam F, and Knowlton N. 2002. Diversity and distribution of coral-associated bacteria. Marine Ecology Progress Series 243:1-10.

Roitman S, Joseph Pollock F, and Medina M. 2018. Coral Microbiomes as Bioindicators of Reef Health. Population Genomics. Cham: Springer.

Rowan R. 2004. Coral bleaching: Thermal adaptation in reef coral symbionts. Nature 430:742-742.

Smith EG, Ketchum RN, and Burt JA. 2017. Host specificity of Symbiodinium variants revealed by an ITS2 metahaplotype approach. ISME J 11:1500-1503.

Spor A, Koren O, and Ley R. 2011. Unravelling the effects of the environment and host genotype on the gut microbiome. Nature Reviews Microbiology 9:279-290.

Sunagawa S, Woodley CM, and Medina Mn. 2010. Threatened corals provide underexplored microbial habitats. PLOS ONE 5:e9554. 
506

507

508

509

510

511

512

513

514

515

516

517

518

519

520

521

522

523

524

525

526

527

528

529

530

531

532

533

534

535

536

Thurber RV, Payet JP, Thurber AR, and Correa AMS. 2017. Virus-host interactions and their roles in coral reef health and disease. Nature Reviews Microbiology 15:205-216.

Turner S, Pryer KM, Miao VP, and Palmer JD. 1999. Investigating deep phylogenetic relationships among cyanobacteria and plastids by small subunit rRNA sequence analysis. Journal of Eukaryotic Microbiology 46:327-338.

VanWoesik R, DeVantier LM, and Glazebrook JS. 1995. Effects of cyclone 'Joy' on nearshore coral communities of the Great Barrier Reef. Marine Ecology Progress Series 128:261-270.

Vega Thurber RL, Willner-Hall D, Rodriguez-Mueller B, Desnues C, Edwards RA, Angly F, Dinsdale EA, Kelly L, and Rohwer F. 2009. Metagenomic analysis of stressed coral holobionts. Environmental Microbiology 11:2148-2163.

Wagner MR, Lundberg DS, Del Rio TG, Tringe SG, Dangl JL, and Mitchell-Olds T. 2016. Host genotype and age shape the leaf and root microbiomes of a wild perennial plant. Nat Commun 7:12151.

Webster NS, and Reusch TBH. 2017. Microbial contributions to the persistence of coral reefs. Isme Journal 11:2167-2174.

Wickham H. 2009. ggplot2: elegant graphics for data analysis: Springer New York.

Wullaert A, Lamkanfi M, and McCoy KD. 2018. Defining the Impact of Host Genotypes on Microbiota Composition Requires Meticulous Control of Experimental Variables. Immunity 48:605-607.

Zaneveld JR, Burkepile DE, Shantz AA, Pritchard CE, McMinds R, Payet JP, RoryWelsh, Correa AMS, Lemoine NP, Rosales S, Fuchs C, Maynard JA, and Thurber RV. 2016. Overfishing and nutrient pollution interact with temperature to disrupt coral reefs down to microbial scales. Nature Communications 7:11833.

Zaneveld JR, McMinds R, and Thurber RV. 2017. Stress and stability: applying the Anna Karenina principle to animal microbiomes. Nature Microbiology 2:17121

Zhang YY, Ling J, Yang QS, Wang YS, Sun CC, Sun HY, Feng JB, Jiang YF, Zhang YZ, Wu ML, and Dong JD. 2015. The diversity of coral associated bacteria and the environmental factors affect their community variation. Ecotoxicology 24:1467-1477.

Ziegler M, Roik A, Porter A, Zubier K, Mudarris MS, Ormond R, and Voolstra CR. 2016. Coral microbial community dynamics in response to anthropogenic impacts near a major city in the central Red Sea. Marine Pollution Bulletin. 105:629-40

Ziegler M, Seneca FO, Yum LK, Palumbi SR, and Voolstra CR. 2017. Bacterial community dynamics are linked to patterns of coral heat tolerance. Nature Communications 8:14213. 


\section{Table $\mathbf{1}$ (on next page)}

Sequencing and sample overview 


\begin{tabular}{cccccl}
\hline Host-genotype & Total no. of samples & No. of sequences & Richness $^{\mathrm{a}}$ & Evenness $^{\mathrm{a}}$ & Shannon Index $^{\mathrm{a}}$ \\
\hline A & 12 & $54,352( \pm 18,259)$ & $71( \pm 64)$ & $0.63( \pm 0.05)$ & $2.53( \pm 0.48)$ \\
B & 12 & $31,702( \pm 19,058)$ & $51( \pm 44)$ & $0.66( \pm 0.14)$ & $2.49( \pm 0.86)$ \\
C & 12 & $26,421( \pm 26,065)$ & $108( \pm 86)$ & $0.73( \pm 0.11)$ & $3.23( \pm 0.65)$ \\
D & 12 & $59,543( \pm 28,560)$ & $101( \pm 102)$ & $0.64( \pm 0.07)$ & $2.74( \pm 0.80)$ \\
E & 12 & $27,348( \pm 24,386)$ & $100( \pm 110)$ & $0.69( \pm 0.10)$ & $2.97( \pm 0.81)$ \\
F & 12 & $36,097( \pm 21,293)$ & $108( \pm 103)$ & $0.73( \pm 0.08)$ & $3.18( \pm 0.84)$ \\
G & 4 & $55,460( \pm 35,822$ & $126( \pm 74)$ & $0.75( \pm 0.07)$ & $3.46( \pm 0.74)$ \\
H & 12 & $44,101( \pm 19,488)$ & $92( \pm 63)$ & $0.65( \pm 0.14)$ & $2.81( \pm 0.64)$ \\
I & 12 & $51,998( \pm 23,968)$ & $109( \pm 73)$ & $0.63( \pm 0.08)$ & $2.82( \pm 0.65)$ \\
\hline
\end{tabular}

a) diversity indices (average \pm SD) for each host genotype are based on a non-rarefied ASV table from which chloroplast and mitochondria derived reads were removed 
Figure $\mathbf{1}$ (on next page)

Conceptual overview of the experimental design.

Acropora tenuis colonies ( $n=9$ ) were fragmented and coral nubbins of each host genotype (A-I) were exposed to three different treatment conditions (control, single stress and cumulative stress) and sampled on a regular basis throughout the experiment (day 1, day 10 , day 14 and day 19). Image credit: Bettina Glasl. 
control

temperature: $27.5^{\circ} \mathrm{C}$ pCO²: 400 ppm

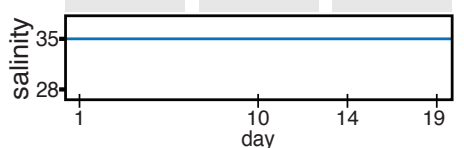

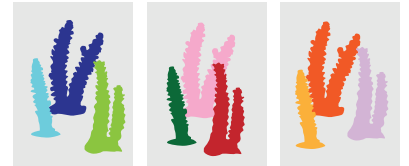

day single stress

temperature: $27.5^{\circ} \mathrm{C}$

pCO: 400 ppm cumulative stress

temperature: $29.5^{\circ} \mathrm{C}$ pCO2: 900 ppm

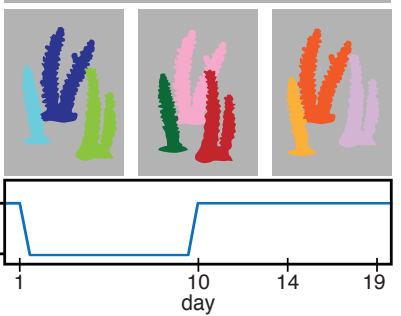


Figure 2 (on next page)

Physiological response of Acropora tenuis under control, single stress and cumulative stress treatments.

Variations in the chlorophyll a ( $\mathrm{Chl}$ a) concentration, protein concentration, effective quantum yield $(\triangle \mathrm{F} / \mathrm{Fm}$ ') and symbiont cell density (Symbiont density) of $A$. tenuis (A) over time (day 1, 10, 14 and 19) and (B) between individual host-genotypes (A-I). Physiological parameters are z-score standardised and error bars represent standard deviations. 
PeerJ

A
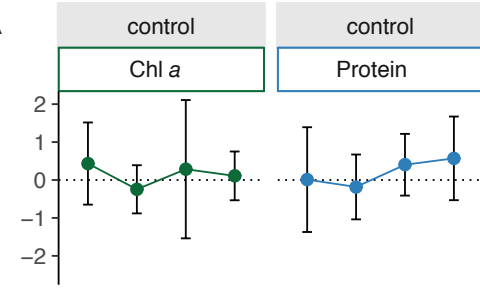

Shl a
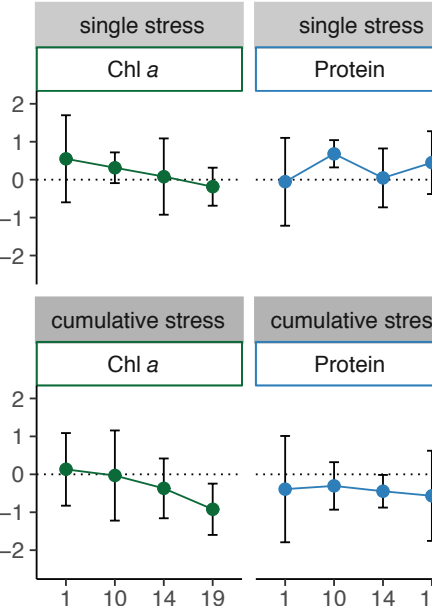

cumulative stress Protein

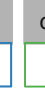

cumulative stress

cumulative stress

Symbiont density $\Delta \mathrm{F} / \mathrm{Fm}$
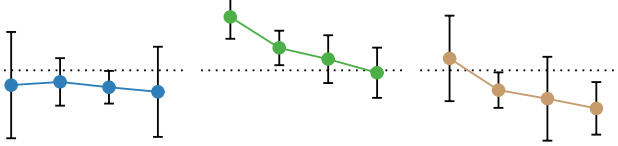

B
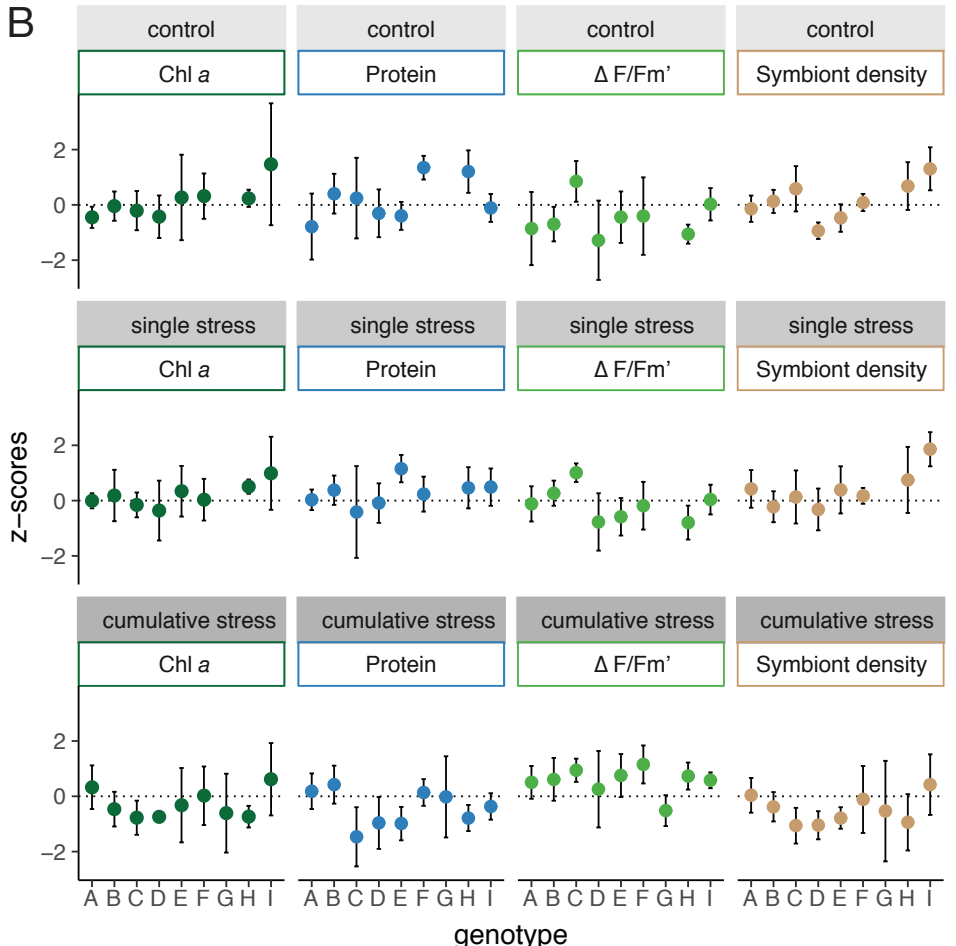
Figure 3 (on next page)

Configurational and compositional stability of Acropora tenuis microbiome.

(A) Multivariate dispersion (heterogeneity) measured by the distance to the group centroid for each host-genotype (A-I) within each treatment (control, acute stress and cumulative stress) over time (day 1, 10, 14 and 19). (B) Non-metric multidimensional scaling (NMDS) illustrating compositional similarity of sample replicates of each host-genotype (A-I) under different treatment conditions (control, single stress and cumulative stress). 
Figure 4 (on next page)

The taxonomic composition of the Acropora tenuis microbiome.

(A)The $A$. tenuis microbiome was dominated by the bacterial genus Endozoicomonas (average relative abundance of $48 \%$ ), with one Endozoicomonas ASV (ASV 11) present in all samples (average relative abundance of $19 \%$ ). (B) The average contribution of the remaining microbiome (others) is displayed as an alluvial diagram, depicting the proportional contribution of bacterial phyla (classes for Proteobacteria). Mean relative abundances (\%) are provided for bacterial taxa $>1 \%$. 


\section{Figure 5 (on next page)}

Composition and distribution of Endozoicomonas assemblages.

(A) Total relative abundance of Endozoicomonas and the relative abundance distribution of the 11 most abundant Endozoicomonas amplicon sequence variants (ASVs) associated with individual coral nubbins of each host genotype (A-I) under control, single stress and cumulative stress conditions over time (day 1, 10, 14 and 19). (B) Distance-based Redundancy Analysis (db-RDA) quantifying the contribution of host-genotype to significantly explaining the observed compositional variation of the Endozoicomonas microbiome. (C) Phylogenetic tree of the 11 most abundant Endozoicomonas ASVs (including the ubiquitously present ASV 11) and their average relative abundance within a host genotype. 


\section{Figure 6 (on next page)}

Microbial indicators significantly associated with one and / or two treatments.

Indicators were identified based on their occurrence and abundance in coral tissue post stress exposure (excluding samples collected at day 1 ) using Indicator Value analysis. Each dot represents a single amplicon sequence variant (ASV), labelled with the taxonomic affiliation and their average relative abundance in the treatment group. 
D_4_Phyllobacteriaceae -

D_4_Vibrionaceae -

D_4_Rhodobacteraceae

D_4_Planctomycetaceae

D_4_Hahellaceae -

D_4_Flavobacteriaceae

D_4_Family XII

D_4_Enterobacteriaceae -

D_4_Cryomorphaceae

D_4_Chitinophagaceae control

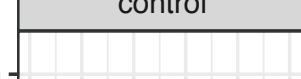

.
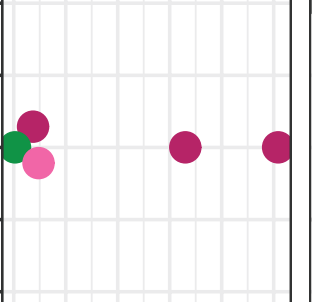

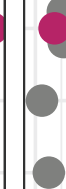
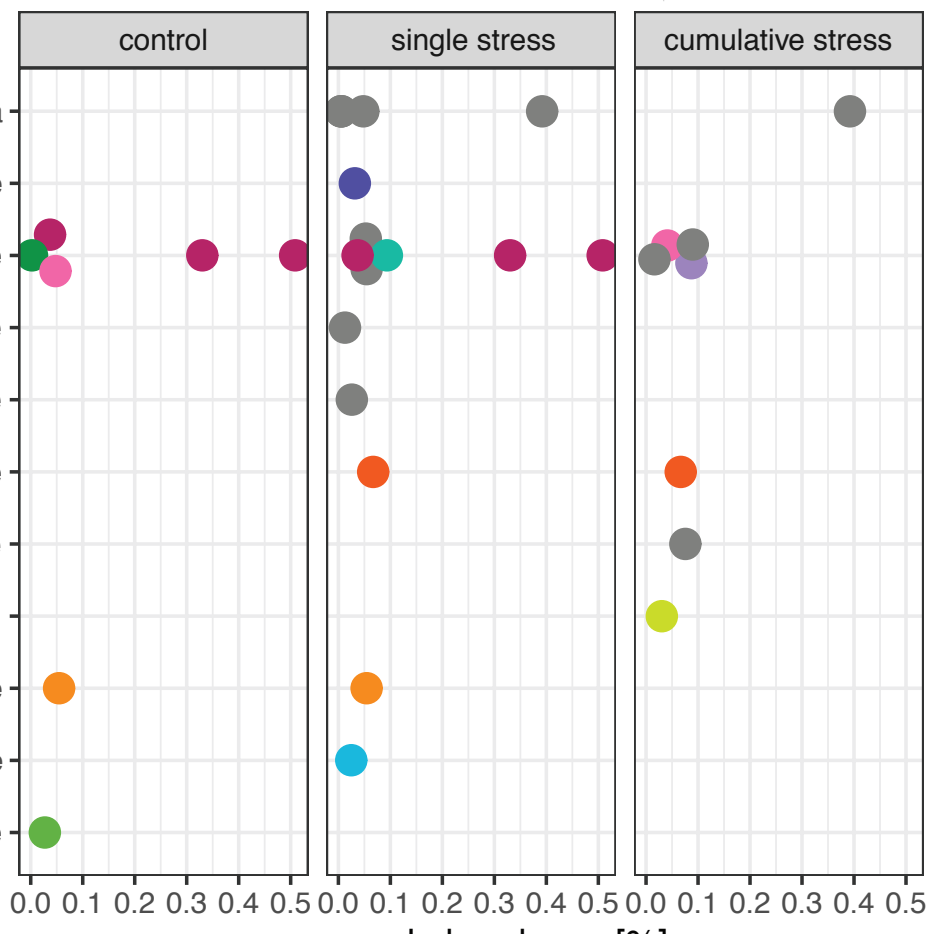

Peer revewing mein

\section{Genus}

D_5_Endozoicomonas

D_5_Escherichia-Shigella

D_5_Fusibacter

D_5_Hydrotalea

D_5_Lentibacter

D_5_Nautella

D_5_Owenweeksia

D_5_Photobacterium

D_5_Roseovarius

D_5_Ruegeria

D_5_uncultured

unidentified 